Provided for non-commercial research and education use. Not for reproduction, distribution or commercial use.

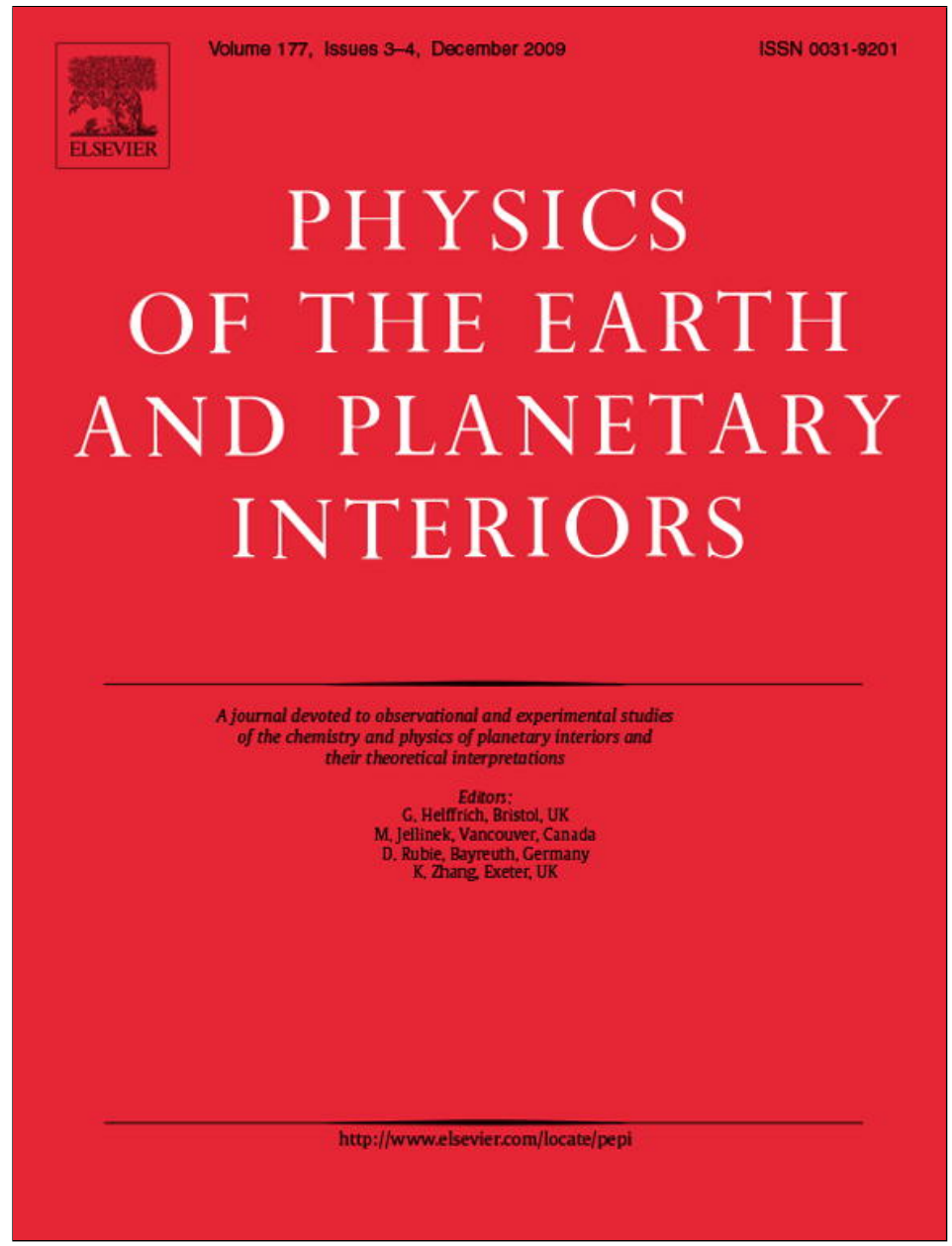

This article appeared in a journal published by Elsevier. The attached copy is furnished to the author for internal non-commercial research and education use, including for instruction at the authors institution and sharing with colleagues.

Other uses, including reproduction and distribution, or selling or licensing copies, or posting to personal, institutional or third party websites are prohibited.

In most cases authors are permitted to post their version of the article (e.g. in Word or Tex form) to their personal website or institutional repository. Authors requiring further information regarding Elsevier's archiving and manuscript policies are encouraged to visit:

http://www.elsevier.com/copyright 


\title{
Effects of anisotropy in geostrophic turbulence
}

\author{
P. Hejda ${ }^{\mathrm{a}}$, M. Reshetnyak ${ }^{\mathrm{b}, *}$ \\ a Institute of Geophysics, Academy of Sciences, 14131 Prague, Czech Republic \\ ${ }^{\mathrm{b}}$ Institute of the Physics of the Earth, Russian Acad. Sci, B.Gruzinskaya 10, 123995 Moscow, Russia
}

\section{A R T I C L E I N F O}

\section{Article history:}

Received 5 March 2009

Received in revised form 2 July 2009

Accepted 19 August 2009

Edited by: K. Zhang

\section{MSC:}

76F65

PACS:

91.25.CW

Keywords:

Liquid core

Thermal convection

Geostrophic balance

Cascade processes

\begin{abstract}
A B S T R A C T
The Boussinesq model of convection in a flat layer with heating from below is considered. We analyze the effects of anisotropy caused by rapid rotation in physical and wave spaces and demonstrate the suppression of energy transfer by rotation. We also examine the structure of the wave triangle in nonlinear interaction. The range of parameters is adapted to the models of convection in the geodynamo.
\end{abstract}

(C) 2009 Elsevier B.V. All rights reserved.

\section{Introduction}

Approximation in the form of homogeneous and isotropic turbulence is quite crude for describing many geophysical applications. Usually, there are two reasons why this approximation can be violated. One of them is a rapid daily rotation characterized by a small Rossby number, Ro $\ll 1$. This case is typical for problems of meteorology and physics of the ocean where its influence is principal (Gill, 1982). However, the most critical regime with an extremely small Rossby number occurs in the liquid planetary cores. There, the Coriolis force is three orders of magnitude larger than the nonlinear term, which changes the balance of the forces in the Navier-Stokes equation and the spectral properties of turbulence for large Reynolds numbers, Re. Another source of anisotropy of convection in the liquid core is a strong magnetic field which, in combination with rotation, leads to the plate-like structure of convective patterns (Braginsky and Meytlis, 1990; Matsushima et al., 1999; Donald and Roberts, 2004). In the present paper we consider the former source of anisotropy related to rapid rotation.
The introduction of rotation leads to substantial rearranging of the flow, both in the physical and in the wave spaces. In spite of this, the Coriolis force itself does not produce work. It can redistribute the energy between the scales and lead to inverse cascades, which is known in direct numerical simulations (DNS) as an increase of the kinetic energy on large scales (Hossain, 1994) predicted in the renormalization group theory (McComb, 1992). As a result, the slope of the spectrum of the kinetic energy changes from $-5 / 3$ to -2 (Zhou, 1995; Constantin, 2002). This change is closely related to the break of the energy transfer over the spectrum (Zhou, 1995).

Let us proceed to the thermal convection problem, when an additional equation of heat transfer leads to the transformation of cellular convection to the cyclonic convection. This problem has been studied thoroughly in the pioneering works on thermal convection (Chandrasekhar, 1961; Roberts, 1965; Busse, 1970), as well as in later papers on the geodynamo (see Rüdiger and Hollerbach, 2004 for references).

It appears that rapid rotation causes a new kind of balance between pressure and the Coriolis force (geostrophic balance) (Pedlosky, 1987): $1_{z} \times \mathbf{V} \sim \nabla p$, which leads to small gradients along the axis of rotation $\mathbf{z}$ : $\frac{\partial \mathbf{V}}{\partial z} \sim 0 .{ }^{1}$ As a result, cyclones and anticy-

\footnotetext{
${ }^{1}$ Note that balance of the potential part of the Coriolis force and the pressure holds.
}

\footnotetext{
Corresponding author. Tel.: +7 9031002308

E-mail addresses: ph@ig.cas.cz (P. Hejda), m.reshetnyak@gmail.com (M. Reshetnyak).
} 0031-9201/\$ - see front matter @ 2009 Elsevier B.V. All rights reserved. doi:10.1016/j.pepi.2009.08.006 
clones prolonged along $\mathbf{z}$ of a small diameter appear. If one takes the parameters of the Earth's liquid core and decreases the amplitude of the heating sources to the onset of convection, the diameter of cyclones will then be $d_{c} \sim E^{1 / 3} L_{z} \approx 10^{-5} L_{z}$, where $E \sim 10^{-15}$ is the Ekman number and $L_{z}$ is the height of the cyclone. In reality, the heat sources are quite larger, and there is a wave packet of such cyclones. However, estimates of the order of the energy indicates that, at least for the first 3-4 orders of the wave number $k$, geostrophic balance takes place. This exceeds the extent of geomagnetic spectra and should be taken into account in geodynamo models. This was already started in the development of the full tensor approach to anisotropic viscosity (Phillips and Ivers, 2003), numerical simulations (Matsushima et al., 1999) and estimates of the anisotropy of heat mass transfer (Donald and Roberts, 2004; Matsui and Buffett, 2005).

The other role of the Coriolis force, which will be the subject of our paper, is to control the energy transfer over the spectrum in the system. Whereas if before the nonlinear term was of the same order with the pressure, which could not block the curl part of the term, then with the Coriolis force the pressure and other potential forces (e.g., the Archemedean force) block the potential part of the nonlinear term. And the rest of the curl parts of the forces and Coriolis force block the nonlinear term. As a result, the spectrum becomes steeper. That is what we have on small scales, $l \ll d_{c}$. At the same time, on scales $l \gg d_{c}$, the system can be in a state of statistical equilibrium: dissipation is negligible, and over the long term the energy exchange between the scales is small. It appears that the estimate of only the spectra of kinetic energy is not enough to resolve the states with and without rotation and intensive heat sources, and one needs to keep track of the fluxes of kinetic energy over the spectrum, which is considered below.

Hereinafter, on the example of an 3D model of thermal convection in a plane layer, we consider the properties of anisotropy caused by rotation both in the physical and especially in the wave spaces started in (Reshetnyak and Hejda, 2008) typical for geodynamo regimes and compare these results with nonrotating convection.

\section{The Boussinesq model}

\subsection{Equations in physical space}

The thermal convection equations for an incompressible fluid $(\nabla \cdot \mathbf{V}=0)$ in a layer of height $L$ rotating with angular velocity $\Omega$ in a Cartesian system of coordinates $(x, y, z)$ in its traditional dimensionless form can be expressed as follows:

$$
\begin{aligned}
& E \operatorname{Pr}^{-1}\left[\frac{\partial \mathbf{V}}{\partial t}+(\mathbf{V} \cdot \nabla) \mathbf{V}\right]=-\nabla P-\mathbf{1}_{\mathbf{z}} \times \mathbf{V}+\operatorname{Ra} T z \mathbf{1}_{\mathbf{z}}+E \Delta \mathbf{V} \\
& \frac{\partial T}{\partial t}+(\mathbf{V} \cdot \nabla)\left(T+T_{0}\right)=\Delta T .
\end{aligned}
$$

Velocity $\mathbf{V}$, pressure $P$ and the typical diffusion time $t$ are measured in units of $\kappa / L, \rho \kappa^{2} / L^{2}$ and $L^{2} / \kappa$, respectively, where $\kappa$ is the thermal diffusivity, $\rho$ is the density, $\operatorname{Pr}=\kappa / \nu$ is the Prandtl number, $E=v /\left(2 \Omega L^{2}\right)$ is the Ekman number, and $v$ is the kinematic viscosity. $\mathrm{Ra}=\left(\alpha g_{0} \delta T L\right) / 2 \Omega \kappa$ is the modified Rayleigh number, $\alpha$ is the coefficient of volume expansion, $\delta T$ is the unit of temperature, for more details see (Jones, 2000), $g_{0}$ is the gravitational acceleration, and $T_{0}=1-z$ is the heating from below. The problem is closed with periodical boundary conditions in the $(x, y)$ plane. In the $z$-direction, we use simplified conditions (Cattaneo et al., 2003): $T=0, V_{z}=\partial V_{x} / \partial z=\partial V_{y} / \partial z=0$ at $z=0,1$.

\subsection{Equations in wave space}

To solve problem (1) we apply the pseudo-spectral approach (Orszag, 1971). We follow the modification of the original approach that has been frequently used in thermal convection and geodynamo: we change FFT in z-direction to the sine-/cosinedecomposition leaving the full FFT in the horizontal directions. This let us to provide the mentioned above boundary conditions in $z$ direction (see also Jones and Roberts, 2000; Buffett, 2003). The equations are solved in the wave space. To calculate the nonlinear terms one needs to apply the inverse Fourier transform, then calculate the product in physical space, apply the Fourier transform of the product, and finally calculate the derivatives in wave space. After eliminating the pressure using the divergence-free condition $\mathbf{k} \cdot \mathbf{V}=0$ we arrive at:

$$
\begin{aligned}
& E\left[\operatorname{Pr}^{-1} \frac{\partial \mathbf{V}}{\partial t}+k^{2} \mathbf{V}\right]_{\mathbf{k}}=\mathbf{k} \mathcal{P}_{\mathbf{k}}+\mathbf{F}_{\mathbf{k}} \\
& {\left[\frac{\partial T}{\partial t}+k^{2} T\right]_{\mathbf{k}}=-\left[(\mathbf{V} \cdot \nabla) T+V_{r}\right]_{\mathbf{k}},}
\end{aligned}
$$

with

$$
\begin{aligned}
& \mathcal{P}_{\mathbf{k}}=-\frac{\mathbf{k} \cdot \mathbf{F}_{\mathbf{k}}}{k^{2}}, \quad k^{2}=k_{\beta} k_{\beta}, \quad \beta=1 \ldots 3 \\
& \mathbf{F}_{\mathbf{k}}=\left[\operatorname{Pr}^{-1} \mathbf{V} \times(\nabla \times \mathbf{V})+\operatorname{RaT} \mathbf{1}_{\mathbf{z}}-\mathbf{1}_{\mathbf{z}} \times \mathbf{V}\right]_{\mathbf{k}} .
\end{aligned}
$$

For integration in time we use the explicit Adams-Bashforth (AB2) scheme for nonlinear terms. The linear terms are treated using the Crank-Nicolson (CN) scheme. To resolve the diffusion terms, we use the well-known trick of changing the variable which helps to increase the time step significantly. Consider equation

$$
\frac{\partial A}{\partial t}+k^{2} A=U
$$

Alter it to read

$\frac{\partial A e^{k^{2} \gamma t}}{\partial t}=U e^{k^{2} \gamma t}$

and then apply the $\mathrm{CN}$ scheme.

The most time-consuming part of our MPI code are the Fast Fourier transforms. To make our code more efficient we use various modifications of known FFT algorithms, which take into account special kinds of symmetry of the fields. The optimal number of processors for the $128^{3}$ grids is $n \sim 50$. Scalability tests have demonstrated even the presence of superacceleration if the number of processors $<n$.

\section{Fluxes in $k$-space}

Now we introduce some diagnostic tools which are very helpful for the further analysis. To analyze the energy transfer in the wave space, we follow Frisch (1995). Let us decompose the physical field $f$ into a sum of low-frequency and high-frequency counterparts: $f(\mathbf{r})=f^{<}(\mathbf{r})+f^{>}(\mathbf{r})$, where

$f^{<}(\mathbf{r})=\sum_{|k| \leq K} \hat{f}_{k} e^{i \mathbf{k r}}, \quad f^{>}(\mathbf{r})=\sum_{|k|>K} \hat{f}_{k} e^{i \mathbf{k r}}$.

For any periodical functions $f$ and $g$ one has the relation (Frisch, 1995):

$$
\left\langle\frac{\partial f}{\partial x}\right\rangle=0, \quad\left\langle\frac{\partial g}{\partial x}\right\rangle=0,\left\langle g \frac{\partial f}{\partial x}\right\rangle=-\left\langle f \frac{\partial g}{\partial x}\right\rangle,
$$

$\left\langle f^{>} g^{<}\right\rangle=0$, 
where

$\langle f(\mathbf{r})\rangle=\mathcal{V}^{-1} \int_{\mathcal{V}} f(\mathbf{r}) \mathrm{d} \mathbf{r}^{3}$,

stands for averaging $f$ over volume $\mathcal{V}$. Multiplying the Navier-Stokes equation by $\mathbf{Z}^{<}$one derives the equation for $E^{<}$in sphere $k<K$ :

$2^{-1} E \operatorname{Pr}^{-1}\left[\frac{\partial\left\langle V_{i}^{<} V_{i}^{<}\right\rangle}{\partial t}+\Pi(K)\right]=\operatorname{Ra}\left\langle T^{<} V_{z}^{<}\right\rangle-E\left\langle\left(\nabla \omega^{<}\right)^{2}\right\rangle$,
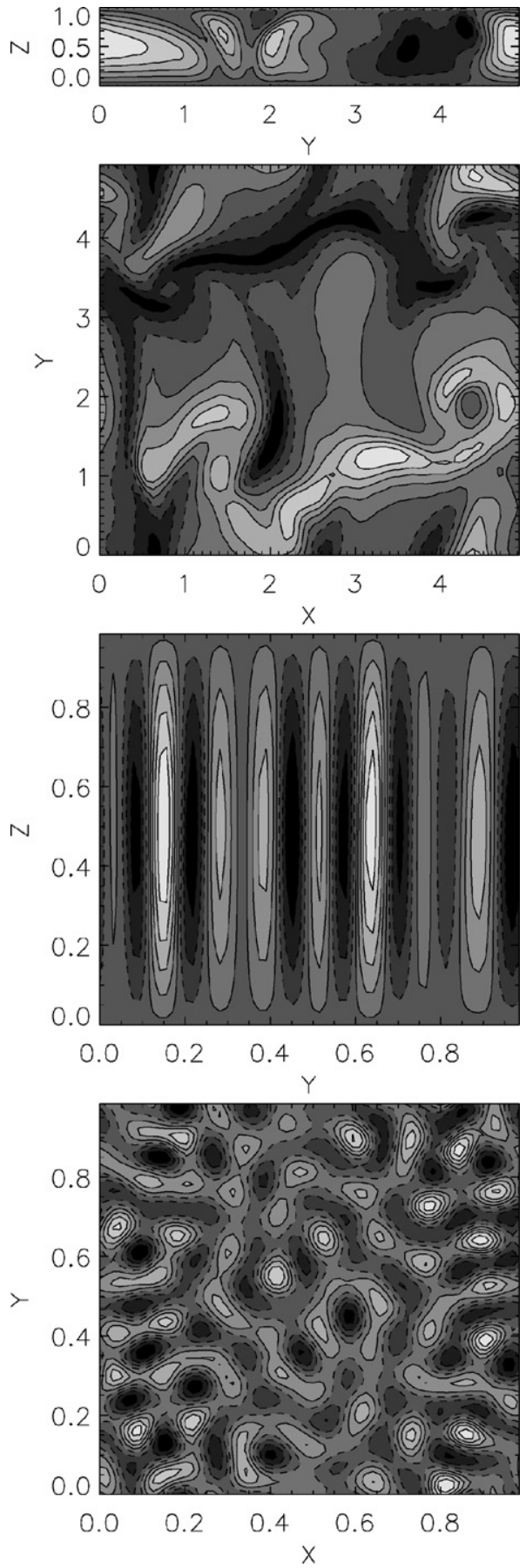

Fig. 1. Sections of the vertical component of velocity, $V_{z}$, without rotation (regime NR, two upper plots) at $x=4.3$, and $z=0.8$. Ranges of the filed: $(-257,506)$ and $(-254,572)$. Two lower plots demonstrate sections of $V_{z}$ with rotation (regime R1) at $x=4.3$ and $z=0.8$. Ranges of the filed: $(-88,127)$ and $(-55,86)$.
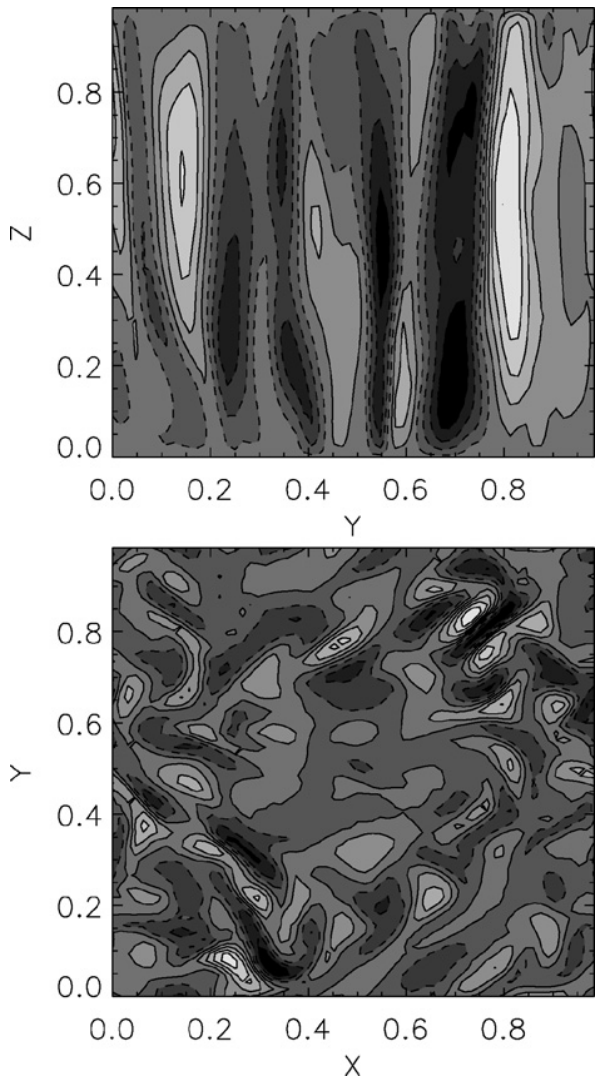

Fig. 2. Sections of the vertical component of velocity $V_{z}$ with rotation (regime R2) at $x=4.3$, and $z=0.8$. Ranges of the filed: $(-407,449)$ and $(-456,600)$

where the integral flux of kinetic energy from the region $k>K$ to $k \leq K$ reads

$\Pi(K)=\left\langle V_{i}^{<} \cdot\left(V_{j} \cdot \nabla_{j}\right) V_{i}\right\rangle$,

with a summation over the repeating indexes $i=1 \ldots 3$. Note, that flux of the Coriolis force is zero: $-\left(\mathbf{V} \times \mathbf{V}^{<}\right)_{z}=-\left(\mathbf{V}^{<} \times \mathbf{V}^{<}\right)_{z}-$ $\left(\mathbf{V}^{>} \times \mathbf{V}^{<}\right)_{z}=0$. The first term in sum is zero due to vector identity and the second due to (5). Introducing the local flux $T_{K}$ :

$T_{K}(k)=-\frac{\partial \Pi(k)}{\partial k}, \quad \int_{k=0}^{\infty} T_{K}(k) \mathrm{d} k=0$,

where we have changed $K$ to $k$, leads to the obvious relation for $E$ in $k$-space:

$\frac{\partial E(k)}{\partial t}=T_{K}(k)+F(k)+D(k)$,

where $E(k)=(1 / 2)(\partial / \partial k)\left\langle\mathbf{V}_{k}^{2}\right\rangle$ is the change of the kinetic energy at $k, F(k)=((\operatorname{RaPr}) / E)(\partial / \partial k)\left\langle T V_{z}^{<}\right\rangle$is the work produced by the Archemedean force, and $D(k)=-\operatorname{Pr}^{2} E(k)$ is the viscous dissipation. Eq. (12) describes the current flux of the kinetic energy through wave number $k$.

To describe a triad interaction mechanism, we can also ask the question: "What is the form of the energy balance equation which describes the transfer of the energy from wave numbers $Q$ and $P$ to $K$ ?" A similar manipulation with the Navier-Stokes equation yields:

$\frac{\partial E(K)}{\partial t}=T_{3}+A(K)+D(K)$,

where $E(K), D(K)$ have the same form as in (12), and $T_{3}=\left\langle V_{i}(K)\right.$. $\left.\left(V_{j}(P) \cdot \nabla_{j}\right) V_{i}(Q)\right\rangle, A(K)=\frac{\operatorname{RaPr}}{E}\left\langle T(K) V_{z}(K)\right\rangle$.

It is also useful to introduce function $T_{2}(K, Q)=\int T_{3}(K, Q, P) \mathrm{d} P$. In general, one needs an explanation. It is possible to show that $T_{2}$ is 

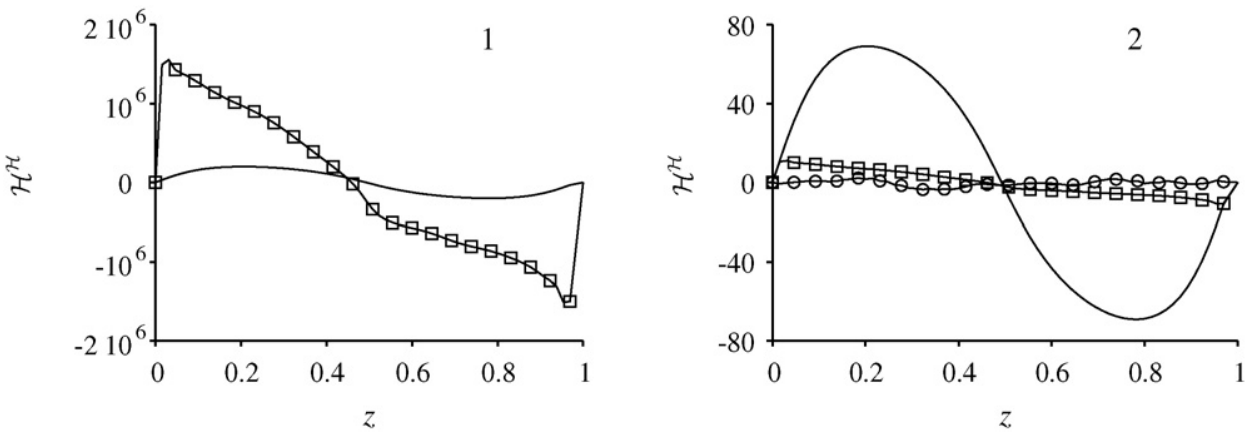

Fig. 3. The profiles of the hydrodynamic helicity $\mathcal{H}^{\mathcal{H}}$ for $E=3 \times 10^{-5}, \operatorname{Pr}=1$, $\operatorname{Ra}=4 \times 10^{2}$ (solid line) and $E=3 \times 10^{-5}$, $\operatorname{Pr}=1, \operatorname{Ra}=1.2 \times 10^{3}$ (squares) $(1$ ). Values are normalized at the mean over the volume kinetic energy $\left(\overline{E_{K}}\right)(2)$. Circles correspond to the regime NR.

the energy flux from harmonic $Q$ to harmonic $K$ (see the references in Alexakis et al., 2007). The analysis of $T_{2}$ allows us to estimate whether the flux is local or not, but not the locality of the interaction itself. The study of $T_{3}$ allow us to recover the full structure of the wave triangle and to explain whether the interaction itself is local. We also note some useful properties of function $T_{2}$ : for the arbitrary periodical (or random homogeneous) nondivergent fields $\mathbf{u}(Q), \mathbf{w}(K)$ and $\mathbf{V}$ one has (Verma, 2004; Alexakis et al., 2005): $T_{2}^{u w}(Q, K)=-T_{2}^{w u}(K, Q)$, where $T_{2}^{u w}(Q, K)=\left\langle u_{i}(K) \cdot\left(V_{j} \cdot \nabla_{j}\right) w_{i}(Q)\right\rangle$, $T_{w u}(Q, K)=\left\langle w_{i}(Q) \cdot\left(V_{j} \cdot \nabla_{j}\right) u_{i}(K)\right\rangle$, which corresponds to the balance of the energy received by shell $K$ from shell $Q$ to that one given by $Q$ to $K$. In the next paragraph, we consider the properties of fluxes $T_{K}, T_{2}, T_{3}$ on an example of model (1) and find how they change when rotation is switched on.

\section{Basic properties of the fields}

The onset of convection in the plane infinite layer is a threshold phenomenon which occurs with the increase of the Rayleigh number to its critical value, $\mathrm{Ra}^{\mathrm{Cr}}$. Here we consider three regimes of convection. We consider simulations without rotation similar to (Meneguzzi and Pouquet, 1989) (but without the magnetic field) and with rotation for two regimes with different amplitudes of the heat sources. All simulations were carried out using a quite rough grid, $N=64^{3}$, which helped us to present the statistics for the $T_{3}$ fluxes. Here are the regimes:

NR: Regime without rotation (the Coriolis term is dropped), ${ }^{2} \mathrm{Ra}=$ $9 \times 10^{5}, \operatorname{Pr}=1, E=1, \operatorname{Re} \sim 700$.

R1: Regime with rotation, $\operatorname{Ra}=4 \times 10^{2}, \operatorname{Pr}=1, E=2 \times 10^{-5}$, $\operatorname{Re} \sim 200$.

R2: Regime with rotation, $\operatorname{Ra}=1 \times 10^{3}, \operatorname{Pr}=1, E=2 \times 10^{-5}$, $\operatorname{Re} \sim 10^{4}$.

Regime NR in Fig. 1 corresponds to turbulent convection without rotation ${ }^{3}$ with a quasi-periodic in time behavior of the kinetic energy $E_{K}(t)$. Note that a small-scale hydrodynamic helicity appears $\mathcal{H}^{\mathcal{H}}=\mathbf{V} \cdot \operatorname{rot} \mathbf{V}$. For the case without rotation, because of the absence of a preferred direction, the mean helicity $\overline{\mathcal{H}_{\mathcal{V}}^{\mathcal{H}}}$ is zero (see Fig. 3 ). One can find the details of the problem in Moffatt (1978); Krause and Rädler (1980); Zeldovich et al. (1983). See also the results of DNS in Meneguzzi and Pouquet (1989).

2 As there is no rotation, Ra and $E$ do not retain their physical meaning defined in Section 2.1. More details on parametrization of nonrotational magnetoconvection can be found in Meneguzzi and Pouquet (1989).

${ }^{3}$ As at the onset of convection the horizontal scales $L_{x}=L_{y}$ of the convective cell are larger than the vertical scale $L_{z}$, the box for simulations is usually longer in $x$-,y-directions. Here $L_{x}=L_{y}=5 L_{z} \equiv 5 L$.
Convection with rotation is characterized by the appearance of numerous vertical rotating columns (cyclones and anticyclones). Their number is defined by the Ekman number as $k_{c} \sim E^{-1 / 3}$ (Chandrasekhar, 1961; Roberts, 1965; Busse, 1970), see also the
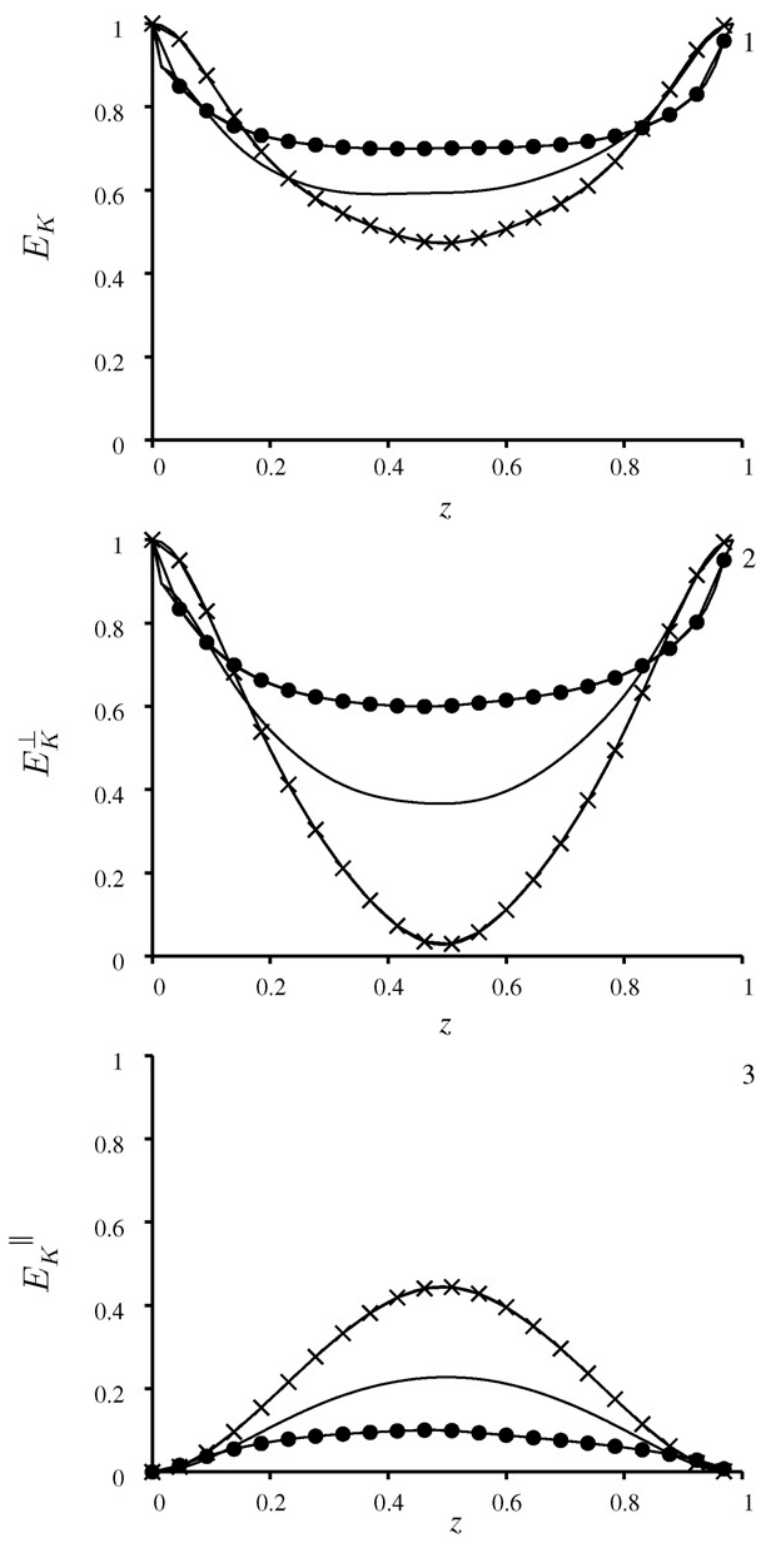

Fig. 4. Dependence of the full kinetic energy $E_{K}$ (1), transverse $E_{K}^{\perp}(2)$ and longitudinal $E_{K}^{\|}$(3) components for regimes NR (solid line), R1 (crosses) and R2 (diamonds). All quantities are normalized at $E_{K}$. 
review by Jones (2000) for details. For $E \ll 1$ the number of cyclones and anticyclones is the same, whereas in the solar-like regimes, where the Coriolis and inertial terms are comparable, the cyclones dominate (Sreenivasan and Davidson, 2008). For the liquid core of the Earth, $E \sim 10^{-15}$. It leads to $k_{c} \sim 10^{5}$ which is still impossible for DNS. Usually one is able to arrive at regimes with $E=10^{-4}$ to $10^{-6}$ (Jones, 2000). The purpose of DNS is to find an asymptotic regime and then to extrapolate the results of the simulations to the terrestrial parameters. The side effect of such cyclonic convection is the decrease of the energy scale of the system and, as a result, the increase of viscous dissipation: the critical Rayleigh number depends on the Ekman number as $\operatorname{Ra}^{\mathrm{Cr}} \sim E^{-1 / 3}$.

Regime R1 corresponds to the geostrophic state near the onset of convection (see Fig. 1). The increase of Ra (regime R2) leads to the
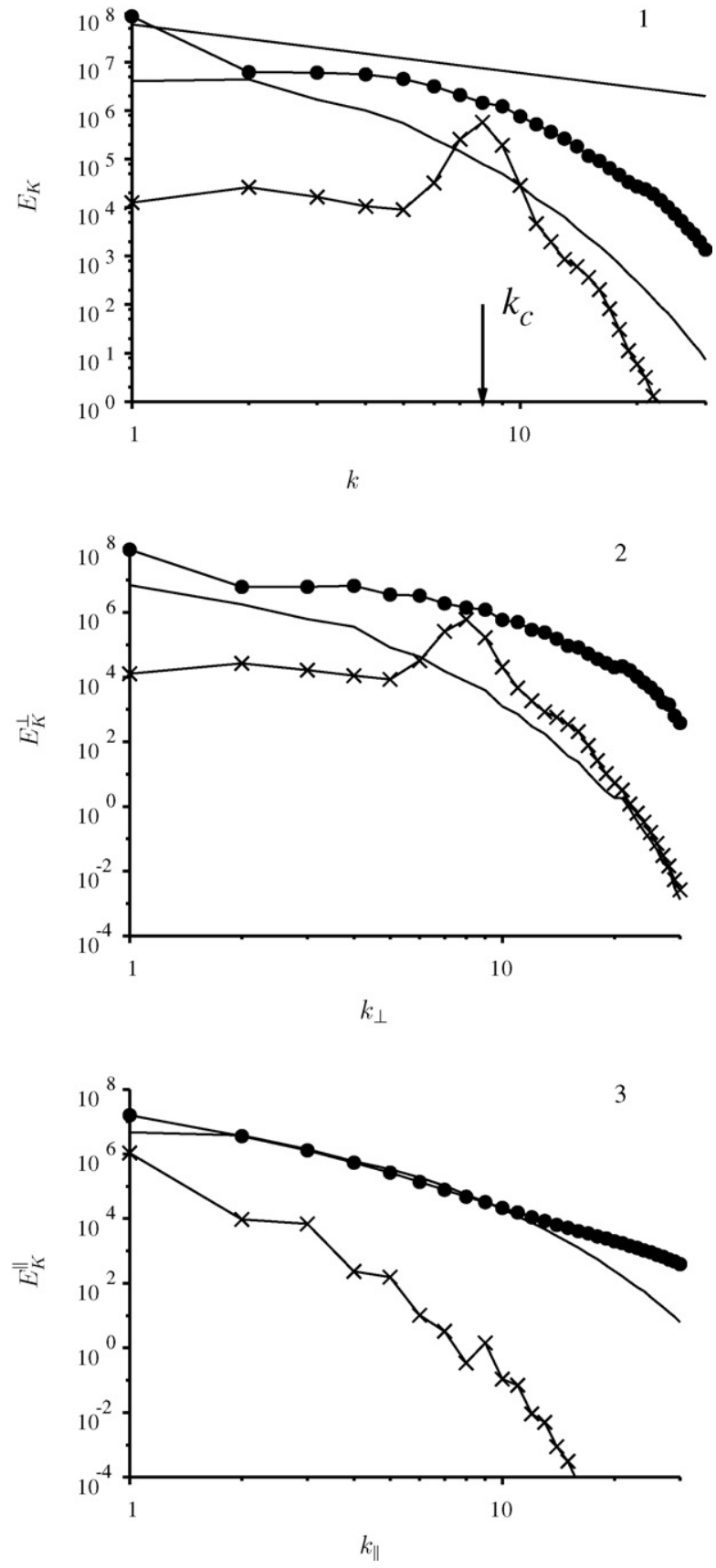

Fig. 5. Spectra of kinetic energy $E_{K}, E_{K}^{\perp}, E_{K}^{\|}$as a function of $k(1), k_{\perp}(2)$ and $k_{\|}$(3) components for regimes NR (solid line), R1 (crosses) and R2 (circles). The straight line corresponds to the Kolmogorov's law of $\sim k^{-5 / 3}$.
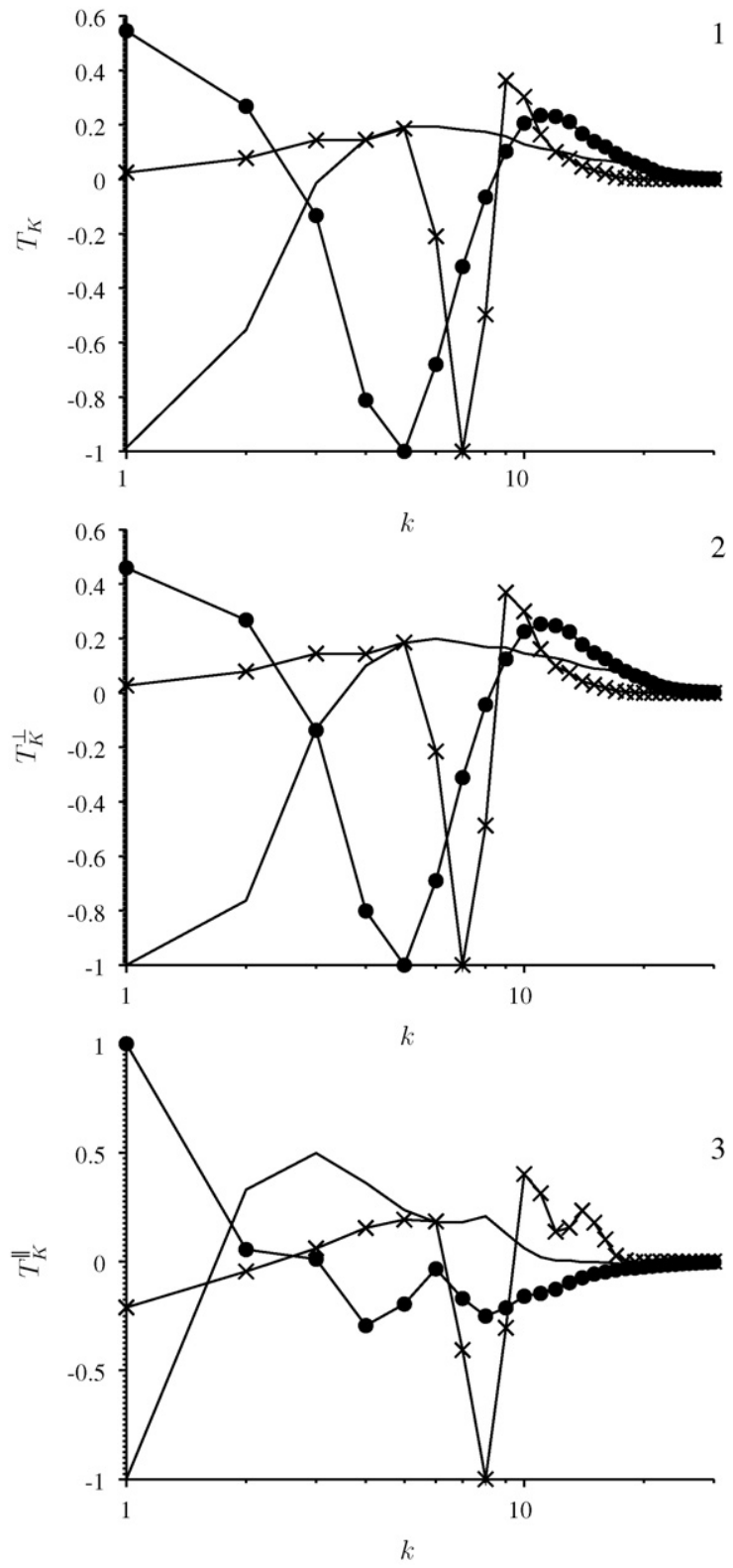

Fig. 6. The fluxes of kinetic energy $T_{K}(1), T_{K}^{\perp}(2), T_{K}^{\|}$(3) as a function of $k$ for regimes NR (solid line), R1 (crosses) and R2 (circles). Functions are normalized at extremums of $T_{K}$.

suppression of the regular cyclones and appearance of the smallscale flows in the $z$-direction, and deviation from the geostrophic state to the so-called quasi-geostrophic turbulence (see Fig. 2). The nonlinear term tends to the amplitude of the Coriolis force and pressure gradient, the temporal behavior of the system becomes chaotic. In both the cases (R1, R2) the mean non-zero helicity generates: $\mathcal{H}^{\mathcal{H}}=\langle\mathbf{V} \cdot \operatorname{rot} \mathbf{V}\rangle_{x y}$ (the average is taken over the $(x, y)$ plane) (see Fig. 3). As was mentioned before, $\mathcal{H}^{\mathcal{H}}$ is zero for regime NR. For $\mathrm{R} 2, \mathcal{H}^{\mathcal{H}}$ is close to the linear function in the main volume.

It is instructive to analyze the behavior of the kinetic energy as a function of depth for different components of the velocity field (see Fig. 4). The different behavior of the transverse $E_{K}^{\perp}=\left(V_{x}^{2}+V_{y}^{2}\right) / 2$ and longitudinal $E_{K}^{\|}=V_{z}^{2} / 2$ components of the energy depends on the different boundary conditions. For the nonrotating case NR, the values of the components are comparable: $\lambda_{\mathrm{NR}}=E_{K}^{\perp} /\left.2 E_{K}^{\|}\right|_{z=0.5} \sim 1$, where the factor 2 appears because of the summation over the two horizontal directions. In contrast, for regime R1 in the vicinity of 
1

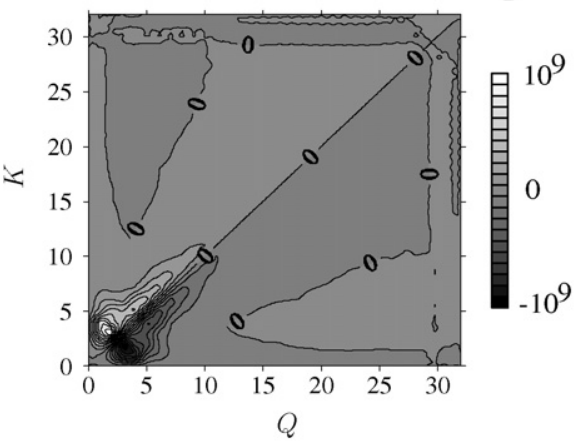

3

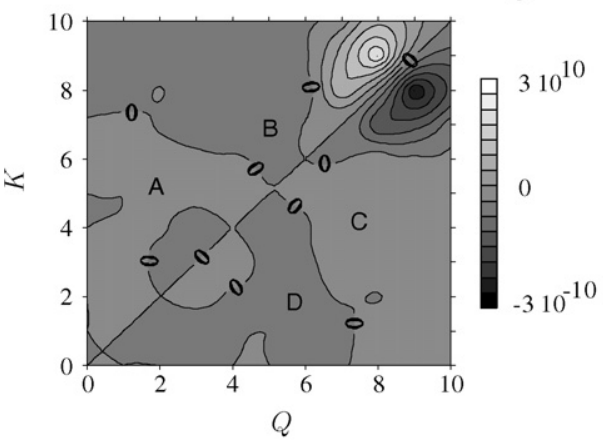

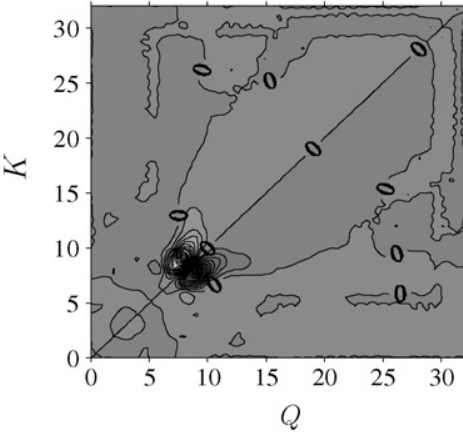

2

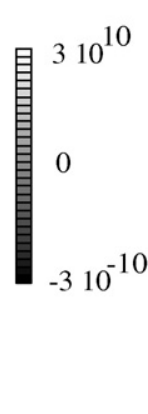

4

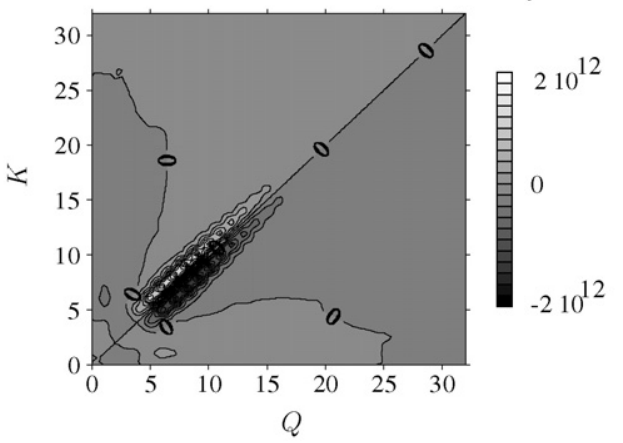

Fig. 7. The fluxes of kinetic energy $T_{2}(k)$ for regimes NR (1), R1 (2 and 3), R2 (4).

the central part of the volume, the transverse velocity changes its sign and, as a result, the ratio increases: $\lambda_{\mathrm{R} 1} \sim 0.1$. This reflects the symmetry property of the flow at the threshold of generation. It is interesting that the increase of Ra (regime R2) leads to reinforcing the anisotropy in the whole volume in the opposite direction: $\lambda_{\mathrm{R} 1} \sim 5$, i.e. at the rather large Ra and under strong rotation, vertical motions are suppressed with rotation. This is the so-called degeneration of three-dimensional convection to the two-dimensional predicted by (Batchelor, 1953), see also the similar degeneration caused by a strong mean magnetic field (Kraichnan, 1965). Also, the increase of $\operatorname{Re}(\mathrm{R} 2)$ goes with the appearance of the layer structures at $z=0,1$. The latter is connected with the formation of a thermal boundary layer, thickness $\delta_{T} \sim \mathrm{Ra}^{-1 / 3}$.

\section{Spectra of the fields and fluxes in the wave space}

Here we continue our analysis of the fluxes in $k$-space performed in Reshetnyak and Hejda (2008) and consider anisotropy effects. For our regimes, we shell consider the integral spectrum of kinetic energy $E_{K}(k)$, as well as its longitudinal $E_{K}^{\|}\left(k_{\|}\right)=\iint E_{K}\left(k_{x}, k_{y}, k_{z}\right) d k_{x} d k_{y}$ and transverse $E_{K}^{\perp}\left(k_{\perp}\right)=$ $\int E_{K}\left(k_{x}, k_{y}, k_{z}\right) d k_{z}$ spectra, where $k_{\perp}^{2}=k_{x}^{2}+k_{y}^{2}, k_{\|} \equiv k_{z}$. For regime NR, the spectral estimates are close to the Kolmogorov dependence $\sim k^{-5 / 3}$ (see Fig. 5). In other words, the spectra for NR are isotropic. It corresponds to the isotropic form of the convective cells at the main scale of the box.

The spectra of convection with rotation differ from those for NR. Regime R1 is close to the onset of convection. The integral spectrum demonstrates a well-pronounced maximum, which corresponds to the scale of cyclones $k_{c} \sim 8$. The increase of Ra (R2) fills the gap for $k<k_{c}$ and the spectrum starts to resemble the spectrum without rotation.

Spectra $E_{K}^{\perp}$ and $E_{K}^{\|}$for R1 are different: spectrum $E_{K}^{\|}$does not feel boundary $k=k_{c}$, whereas $E_{K}^{\perp}$ at $k<k_{c}$ is close to the white noise, and at large $k_{\perp}$ the spectrum decays. For larger Ra, $E_{K}^{\|}$tends to Kolmogorov's asymptotic, and the transverse spectrum is still white for small $k$ and close to the one without rotation at $k_{\perp}>$ $k_{c}$.

Note that for R1 and R2 the form of the integral spectrum $E_{K}$ is defined by the form of its transverse component $E_{K}^{\perp}$.

The apparent similarity in the spectra for NR and R2 does not mean that the physics of the processes are similar. In this respect we remind the reader that the two-dimensional turbulence with inverse cascades and the three-dimensional turbulence with direct cascades have the same slope of " $-5 / 3$ " for the kinetic energy spectrum (Kraichnan and Montgomery, 1980). However, the directions of the energy transfer through the spectrum in these two examples are opposite.

Fig. 6 shows the fluxes of the kinetic $T_{K}$ energies for the regimes mentioned above. At first we consider the integral over all directions. Regime NR for $T_{K}$ demonstrates the well-known behavior for the direct Kolmogorov cascade in 3D. For large scales $T_{K}<0$, these scales are donors and provide energy to the system. On the other hand, the harmonics with large $k$ absorb energy. The two-dimensional turbulence exhibits mirror-symmetrical behavior relative to the axis of the abscissa (Kraichnan and Montgomery, 1980). In this case the energy cascade is inverse.

Rotation essentially changes the behavior of the fluxes of kinetic energy. The leading order wave number is $k_{c}$. For $k>k_{c}$ we also observe the direct cascade of energy $T_{K}>0$. The maximum of $T_{K}$ is shifted relative to the maximum of the energy to large $k$; the larger Re, the stronger the shift. For $k<k_{c}$, the behavior is more complex: for small $k$, the inverse cascade of kinetic energy takes place, $T_{K}>0$. It is very tempting to associate this small region with the appearance of coherent structures (see Tabeling, 2002). On the other hand, for the larger region of $k\left(0 \ldots k_{c}\right)$ we still have the direct cascade $T_{K}<0$. The increase of Re leads to the narrowing of the region with the inverse cascade and to the increase of the inverse flux. One may suggest that the change of the sign of flux $T_{K}$ at $k<k_{c}$ is connected with the appearance of the nonlocal energy transfer: so that the energy to the large-scales $\mathbf{k}_{\mathbf{1}}$ comes from modes $\left|\mathbf{k}_{\mathbf{2}}\right| \sim\left|\mathbf{k}_{\mathbf{3}}\right| \gg\left|\mathbf{k}_{\mathbf{1}}\right|$, 
$\mathbf{k}_{\mathbf{1}}=\mathbf{k}_{\mathbf{2}}+\mathbf{k}_{\mathbf{3}}$ (Waleffe, 1992). Hence, in the case of rotation, two cascades of kinetic energy (direct and inverse) take place simultaneously. As shown in the simulations for the higher resolution (Reshetnyak and Hejda, 2008) these results do not depend on the presence of the magnetic field, if the kinetic and magnetic energies are comparable in order of magnitude.

To estimate the anisotropy of the fluxes, we decompose $T_{K}$ into the sum of the transverse $T_{K}^{\perp}$ and longitudinal $T_{K}^{\|}$parts, so that in Eq. (10) the summation over index $j$ was used for $j=1 \ldots 2$ and $j=3$, respectively. For all three regimes, the fluxes in the $z$-direction are much smaller than in the horizontal plane, however, the form of $T_{K}^{\|}$are similar to that of $T_{K}$. This asymmetry can be explained as follows. Obviously, flux $\Pi_{K}^{\|} \sim V_{z}^{<} \partial E_{K} / \partial z$ is small for geostrophic flows. For NR, it is also small because the kinetic energy does not change too much along the length of the plumes in the z-direction in the main volume. As regards the boundaries $z=0,1, V_{z}$ is small there. That is why the main contribution to $\Pi_{K}$ yields the transverse flux: $\Pi_{K}^{\perp} \sim \mathbf{V}_{\perp}^{<} \cdot \nabla_{\perp} E_{K}$. This asymmetry distinguishes all three regimes from the homogeneous, isotropic models.

\section{Locality of the energy transfer}

Let us consider the structure of the triad interactions. Fig. 7 is a diagram of the antisymmetric (with respect to the diagonal $K=Q$ ) fluxes $T_{2}$ for regimes NR, R1, R2. For NR, the results are similar to the case with the imposed force in Alexakis et al. (2007): harmonics with $K>Q$ take the energy from harmonics with $K<Q$ (direct cascade of the energy). The maximum of the energy flux corresponds to the closest to the diagonal harmonics with $K \sim Q$, i.e. the local energy transfer exists. Note that there are regions (i.e. $Q=5, K=20$ ), in which there is a nonlocal inverse cascade of energy. Omitting these details, the whole behavior is close to the idealized Kolmogorov scenario. As a matter of convenience, we present the diagrams as a function of $K-Q$ in Fig. 8. This clearly demonstrates the existence of the direct cascade, as well as the local interactions with the local energy transfer.

The rotation changes the behavior of $T_{2}$ at $k<k_{c}$, leaving it unchanged at $k>k_{c}$. We shall consider it in more detail. The positions of the extrema are near to $k_{c}$, where the energy transfers to the region of $k$ larger than the leading mode $k_{c}$. On the other hand, at $k<k_{c}$ the flux decreases, which corresponds to the approach to the state of statistical equilibrium, observed in Fig. 6. On the larger scale (see Fig. 7(3)) we resolve the finer structure at small $k$ : region $A$ with a direct cascade of energy, but with an equiprobable energy transfer from the small $Q \sim K$, as well as from the rather large $Q \sim 4 K$. Region $B$ with an inverse cascade (as well as in region $A$ with the weak energy flux of the order of $1 / 10$ of its maximal absolute value) has an elongated strip-like form from $Q \sim K$ to $Q \sim 10 K$. This corresponds to the occurrence of the small negative minimum at $K>Q^{4}$

The increase of Ra (regime R2) leads to the shift of the region with the inverse cascade in the direction of the small $Q$ and $K>Q$. As before, we observe the equilibrium state at $k<k_{c}$. This regime exhibits a longer interval of the wave numbers at $k>k_{c}$ with local energy transfer and the direct cascade. The relative input of the region with the inverse cascade becomes smaller, see Fig. 8(3) and at the same time this input shifts to the large-scale field at $k \ll$ $k_{c}$, which can be interesting for geodynamo applications, where $k_{c} \sim 10^{5}$ and the geomagnetic field generation regions $\left(k \sim 1\right.$ to $10^{3}$ for the typical magnetic Reynolds number $R_{m} \sim 10^{2}$ to $10^{3}$ ) are separated at least by some orders of magnitude of $k$.

${ }^{4}$ We do not consider the behavior of function $T_{2}$ at $K<Q$ (regions $C$ and $\mathrm{D}$ ), because of its antisymmetry.
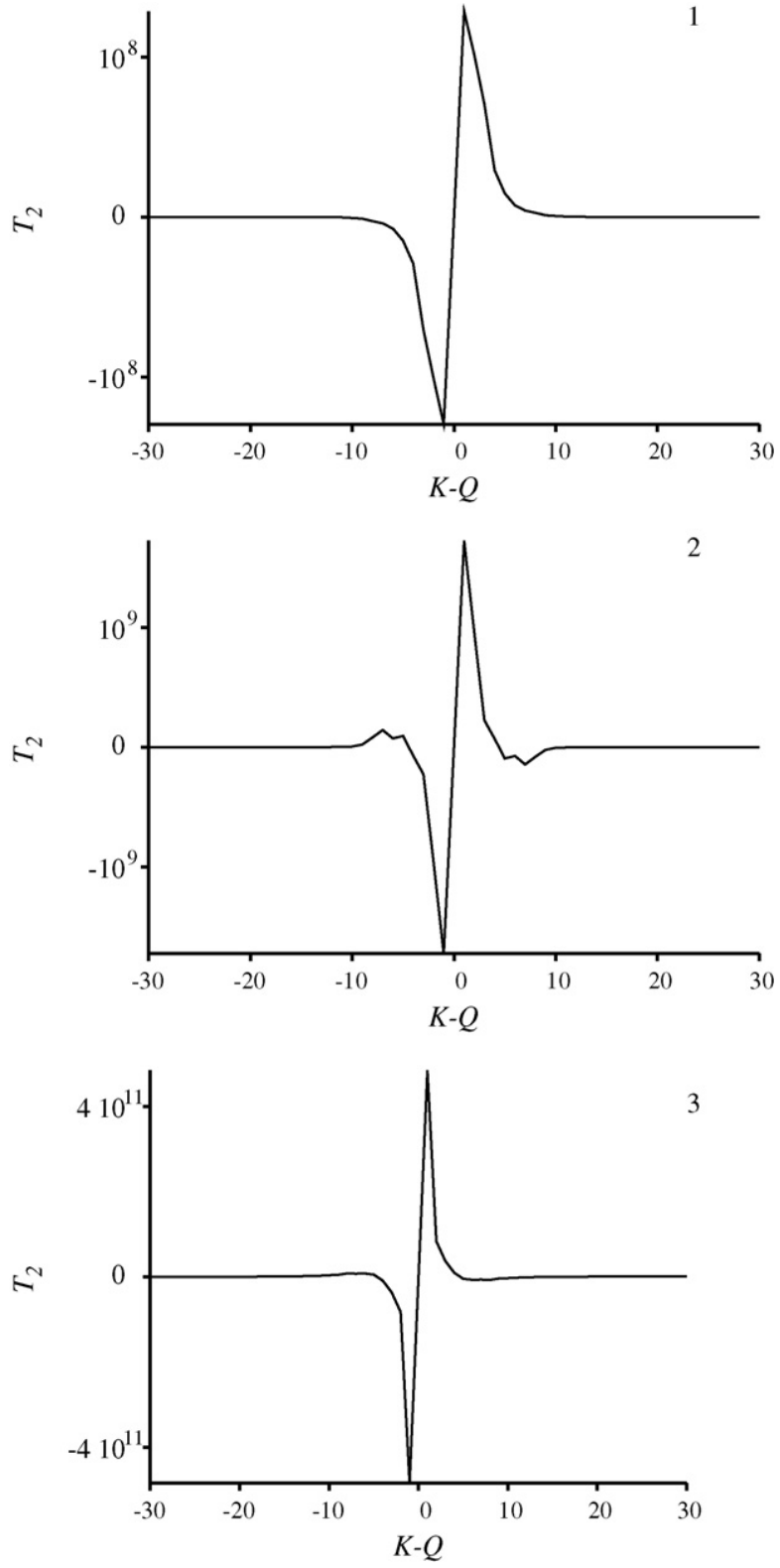

Fig. 8. The fluxes of kinetic energy $T_{2}(K-Q)$ for regimes NR (1), R1 (2), R2 (3).

\section{Locality of interactions}

Let us consider the properties of function $T_{3}$ for three regimes in more details. Because of the symmetry of the problem, we expect that $T_{3}(K, P, Q)=T_{3}(K, Q, P)$, which was used in constructing the discrete analog of the operator. The case without rotation, see Fig. 9(1,2) demonstrates a quite interesting result: the largest input to the energy flux for mode $K$ comes from two sources: from the $P \lesssim K, Q \ll P$ and $Q \lesssim K, P \ll Q$. In other words the wave triangle $(K, P, Q)$ is isosceles with a small angle between the equal sides $\angle(K, P)$ or $\angle(K, Q)$. Taking into account that, according to Fig. 8 , the energy transfers to $K$ from the closest smaller wave number, we come to conclusion that the third small vector is a catalyzer in the interaction while participating in the interaction this highfrequency wave does not provide to the wave $K$ itself with energy. This result is beyond the classical Kolmogorov scenario, according which the wave triangle is an equilateral, i.e. not only the energy transfer is local, but the interaction as well. There are two reasons for this discrepancy. Having in mind the results of Alexakis 

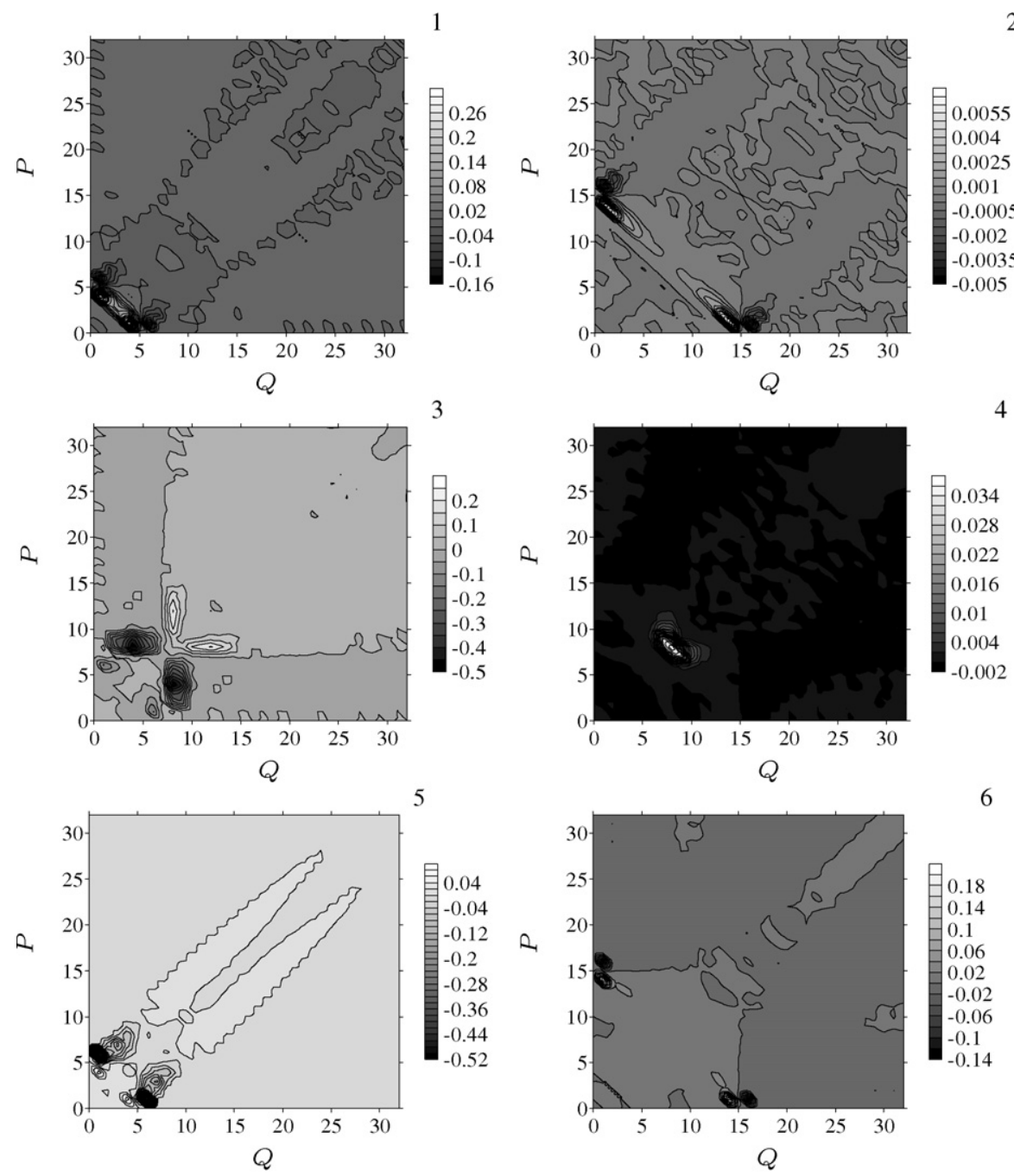

Fig. 9. Fluxes $T_{3}$ for fixed $K$. NR: $K=5$ (graph 1), $K=15(2)$, R1: $K=7(3), K=15(4), \mathrm{R} 2: K=5(5), K=15(6)$ normalized at the maximal values for all $P$, $Q$ and $K$.

et al. (2007), we connect this result with a shortness of the considered spectra. On the other hand, it can be caused by anisotropy concerned with the gravity force. In this connection, it is known that, even for the larger Reynolds numbers in the problem with the imposed external force, the anisotropy on small scales can be substantial (Zhou and Yeung, 1996).

Our modeling demonstrates that there is a strong correlation of the fluxes on small scales with a buoyancy force on main scale. For this purpose, we introduce function $r(K)=\int T_{3}$. $\max (P / Q, Q / P) d P d Q / \int T_{3} d P d Q(P, Q>0)$ (see Fig. 10). Virtually, for all $K$ the angle $\alpha(K)=\int f \cdot T_{3} d P d Q / \int T_{3} d P d Q\left(f=\left(P^{2}+Q^{2}-\right.\right.$

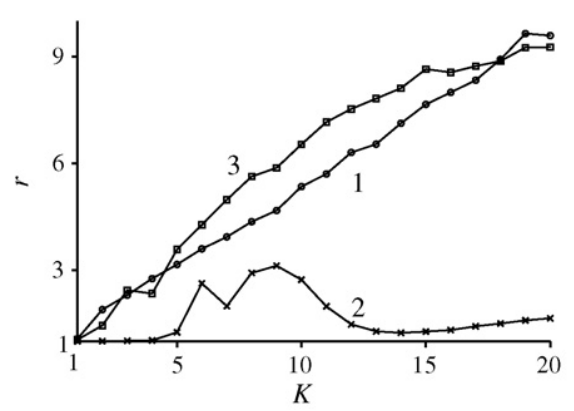

$\left.K^{2}\right) / 2 P Q$ ) between vectors $\mathbf{p}$ and $\mathbf{q}$ is about $100^{\circ}$, i.e. the modes, which take part in the interaction, are orthogonal in the wave space. In the case with rotation the structure of the triangle with small Ra differs from the case without rotation. The particular wave interacts with the set of the waves-see the cross-structure of the diagram in Fig. 10. This can be done by increasing the angle between vectors $\mathbf{p}$ and $\mathbf{q}$. Note that $P \sim Q$ for small $K$ and $P$ (see Fig. 10).

There is a well-pronounced inverse cascade of energy for small $K$, see Fig. 9: harmonic $K=7$ receives energy from the higher wave numbers, transferring the energy, in its turn, to shorter waves. For the larger $K$, there is a state with $P \sim Q$ and $\alpha \sim 100^{\circ}$. In contrast to

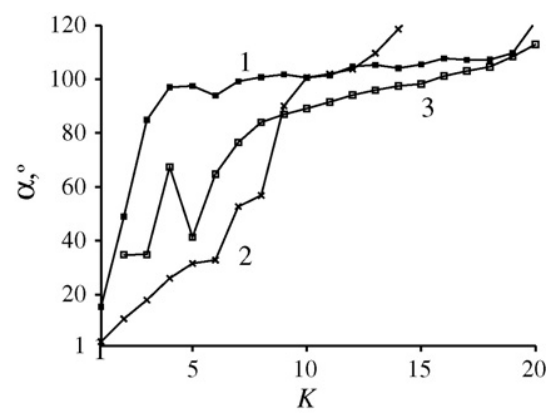

Fig. 10. Dependence maximum of the ratio of two wave numbers $P / Q, Q / P$ (left plot) and angle between vectors $\mathbf{p}$ and $\mathbf{q}$ (right plot), as a function of the resulting harmonic $K$ for regimes NR (solid line), R1 (crosses) and R2 (diamonds). 
the case without rotation, the energy input to $K$ is made by waves $P \sim Q \approx 0.7 \mathrm{~K}$. The increase of Ra leads to the intermediate state.

\section{Conclusion}

To summarize the main results of the paper: the rotation sufficiently changes the structure of the flows in the physical space and its spectral properties. The introduction of rotation leads to the transformation of the typical Rayleigh-Benar cellular convection to cyclonic form. In general, the rotation suppresses convection due to enhanced dissipation: the horizontal scale of the cyclone is $E^{-1 / 3}$ times smaller than its vertical scale $L_{z}$. Moreover, the rotation leads to the selective suppression of the motion along the axis of rotation $\left(\lambda_{\mathrm{R} 2} \ll 1\right)$ and it also upsets the mirror-reflecting symmetry of the system, which is reflected in the non-zero mean hydrodynamic helicity $\overline{\mathcal{H}^{\mathcal{H}}} \neq 0$.

The behavior of the system in the regime with rotation with moderate Ra is very different for $k>k_{c}$ and $k<k_{c}$. For $k<k_{c}$ there is a weak inverse cascade with nonlocal interactions. It is possible to speak of statistical equilibrium when the energy exchange between the Fourier modes is absent. For $k>k_{c}$, the cascade is direct, however, the nonlocal flux from small $k \sim k_{c}$ exists. The slopes of the spectra for $k<k_{c}$ and $k>k_{c}$ are different. For long waves, the spectrum is close to white noise, and for large $k$, the spectrum decays as $k^{-3}$.

We have demonstrated that, even in a pure hydrodynamic system without a magnetic field, a variety of different interactions occurs in the Fourier space. This analysis could be used for adjusting semi-empirical turbulent models proposed for geodynamo purposes in the future. In such models, there should be agreement of the spectra and fluxes in the wave space for scales larger than the cut-off scale $d_{a}$ with DNS of finer resolution. The nontrivial point here is the reproduction of the inverse cascades with $d_{c}<d_{a}$.

\section{References}

Alexakis, A., Mininni, P.D., Pouquet, A., 2005. Shell to shell energy transfer in MHD. I. Steady state turbulence. Phys. Rev. E 72, 046301-046309.

Alexakis, A., Mininni, P.D., Pouquet, A., 2007. Turbulent cascades, transfer, and scale interactions in magnetohydrodynamics. New J. Phys. 298 (9), 1-20.

Batchelor, G.K., 1953. The Theory of Homogeneous Turbulence. Cambridge University Press, Cambridge.

Braginsky, S.I., Meytlis, V.P., 1990. Local turbulence in the Earth's core. Geophys. Astrophys. Fluid Dynam. 55, 71-87.

Buffett, B., 2003. A comparison of subgrid-scale models for large-eddy simulations of convection in the Earth's core. Geophys. J. Int. 153, 753-765.

Busse, F.H., 1970. Thermal instabilities in rapidly rotating systems. J. Fluid Mech. 44 $441-460$
Cattaneo, F., Emonet, T., Weiss, N., 2003. On the interaction between convection and magnetic fields. ApJ. 588, 1183-1198.

Chandrasekhar, S., 1961. Hydrodynamic and Hydromagnetic Stability. Dover Publications. Inc., NY.

Constantin, P., 2002. Energy spectrum of quasigeostrophic turbulence. Phys. Rev. Lett. 89 (18), 184501-184504.

Donald, J.T., Roberts, P.H., 2004. The effect of anisotropic heat transport in the Earth's core on the geodynamo. Geophys. Astrophys. Fluid Dynam. 98 (5) 367-384.

Frisch, U., 1995. Turbulence: The Legacy of A. N. Kolmogorov. Cambridge University Press, Cambridge.

Gill, A.E., 1982. Atmosphere-ocean Dynamics. Academic Press, NY.

Hossain, M., 1994. Reduction of the dimensionality of turbulence due to a strong rotation. Phys. Fluids 6 (4), 1077-1080.

Jones, C.A., 2000. Convection-driven geodynamo models. Phil. Trans. R. Soc. London A 358, 873-897.

Jones, C.A., Roberts, P.H., 2000. Convection driven dynamos in a rotating plane layer J. Fluid Mech. 404, 311-343.

Kraichnan, R.H., 1965. Inertial-range spectrum of hydromagnetic turbulence. Phys. Fluids 8, 1385-1387.

Kraichnan, R.H., Montgomery, D., 1980. Two-dimensional turbulence. Rep. Prog. Phys. 43, 547-619.

Krause, F., Rädler, K.-H., 1980. Mean Field Magnetohydrodynamics and Dynamo Theory. Akademie-Verlag, Berlin.

Matsui, H., Buffett, B., 2005. Sub-grid scale model for convection-driven dynamo in a rotating plane layer. Phys. Earth Planet. Inter. 153, 108-123.

Matsushima, M., Nakajima, T., Roberts, P.H., 1999. The anisotropy of local turbulence in the Earth's core. Earth Planets Space 51, 277-286.

McComb, W.D., 1992. The Physics of Fluid Turbulence. Clarendon Press, Oxford.

Meneguzzi, M., Pouquet, A., 1989. Turbulent dynamos driven by convection. J. Fluid Mech. 205, 297-318.

Moffatt, H.K., 1978. Magnetic Field Generation in Electrically Conducting Fluids. Cambridge University Press, Cambridge.

Orszag, S.A., 1971. Numerical simulation of incompressible flows within simple boundaries. I. Galerkin (spectral) representations. Stud. Appl. Math. L. 4 293-327.

Pedlosky, J., 1987. Geophys. Fluid Dynam. Springer-Verlag, NY.

Phillips, C.G., Ivers, D.J. 2003. Strong field anisotropic diffusion models for the Earth's core. Phys. Earth Planet Int. 140, 13-28.

Reshetnyak, M., Hejda, P., 2008. Direct and inverse cascades in the geodynamo. Nonlin. Process. Geophys. 15, 873-880.

Roberts, P.H., 1965. On the thermal instability of a highly rotating fluid sphere. Astrophys. J. 141, 240-250.

Rüdiger, G., Hollerbach, R., 2004. The Magnetic Universe. Wiley-VCH Verlag GmbH \& Co. KGaA, Weinheim.

Sreenivasan, B., Davidson, P.A., 2008. On the formation of cyclones and anticyclones in a rotating fluid. Phys. Fluids 20, 085104-1-08510411-08510411.

Tabeling, P., 2002. Two-dimensional turbulence: a physicist approach. Phys. Rep. 362, 1-62.

Verma, M., 2004. Statistical theory of magnetohydrodynamic turbulence: recent results. Phys. Rep. 401, 229-380.

Waleffe, F., 1992. The nature of triad interactions in homogeneous turbulence. Phys Fluids A4 (2), 350-363.

Zeldovich, Ya.B., Ruzmaikin, A.A., Sokoloff, D.D., 1983. Magnetic Fields in Astrophysics. Gordon and Breach, NY.

Zhou, Y., 1995. A phenomenological treatment of rotating turbulence. Phys. Fluids 7 (8), 2092-2094.

Zhou, Y., Yeung, P.K., 1996. Scale disparity and spectral transfer in anisotropic numerical turbulence. Phys. Rev. E 53, 1261-1264. 